\title{
AVALIAÇÃO DA INTENSIDADE DE TRÁFEGO E CARGA DE UM FORWARDER SOBRE A COMPACTAÇÃO DE UM LATOSSOLO VERMELHO-AMARELO ${ }^{1}$
}

\author{
Arystides Resende Silva², Moacir de Souza Dias Junior ${ }^{3}$ e Fernando Palha Leite ${ }^{4}$
}

\begin{abstract}
RESUMO - A operação de baldeio florestal está relacionada ao uso de máquinas, as quais trafegam numa mesma linha várias vezes ou aleatoriamente, o que pode causar compactação do solo, alterando o meio onde o sistema radicular se desenvolve e reduzindo a produtividade. Além do número de passadas, a carga de madeira transportada pode afetar a compactação do solo quando as pressões aplicadas pelas máquinas excederem a capacidade de suporte de carga desse solo. Os objetivos deste estudo foram: a) propor modelo de capacidade de suporte de carga para Latossolo Vermelho-Amarelo no Município de Santa Maria de Itabira, MG, em razão da pressão de pré-consolidação e da umidade; e b) determinar, com o uso deste modelo, o efeito da intensidade de tráfego e da carga de Forwarder sobre a estrutura do solo. Para a obtenção dos modelos de capacidade de suporte de carga, 20 amostras indeformadas de solo foram coletadas nas profundidades de 0-3 e 10-13 cm no local onde não houve tráfego. Foram também coletadas 10 amostras indeformadas em cada profundidade, onde o Forwarder trafegou duas, quatro e oito vezes e no local onde o Forwarder trafegou quatro vezes com 1/3 $\left(3 \mathrm{~m}^{3}\right)$, 2/3 $\left(6 \mathrm{~m}^{3}\right)$ e 3/3 $\left(9 \mathrm{~m}^{3}\right)$ de sua carga. As amostras indeformadas foram utilizadas nos ensaios de compressão uniaxial. Determinaram-se também a textura, o teor de matéria orgânica e a densidade de partículas e densidade dos solos. O modelo de capacidade de suporte de carga do LVA é expresso pela equação $\sigma_{\mathrm{p}}=10^{(2,71-1,36 \mathrm{U})}$. Todas as intensidades de tráfego causaram compactação no solo nas duas profundidades estudadas, sendo quatro passadas as que causaram maior compactação do solo, e, à medida que a carga do Forwarder aumentou, a compactação do solo também aumentou nas duas profundidades.
\end{abstract}

Palavras-chave: Compactação do solo, Pressão de pré-consolidação e Mecanização florestal.

\section{EVALUATION OF TRAFFIC INTENSITY AND LOAD OF A FORWARDER ON STRUCTURE OF A RED-YELLOW LATOSOL}

\begin{abstract}
The operation of forest traffic is related to use of machines that pass through the same line repeatedly or randomly, which may cause soil compaction, changing the environment of root growth system development and reducing the productivity. Besides the number of passes, the wood load carried may also affect soil compaction, when the applied pressures by machines exceed soil load support capacity. The objectives of this study were: a) to propose a load support capacity model for a Oxisol (Red-Yellow Latosol, LVA) as a function of preconsolidation pressure and moisture content; $b$ ) to determine, through the model, the effect of traveling and load intensity of a forwarder on the soil structure. To obtain the load support capacity models, 20 undisturbed soil samples were collected in the depth of $0-3$ and $10-13 \mathrm{~cm}$, at a site where there was no traffic. Also 10 undisturbed soil samples were collected for each depth where the Forwarder passed though 2, 4 and 8 times, and where the Forwarder passed in the same line 4 times with 1/3 $\left(3 \mathrm{~m}^{3}\right), 2 / 3\left(6 \mathrm{~m}^{3}\right) e$ $3 / 3\left(9 \mathrm{~m}^{3}\right)$ of its load. The undisturbed soil samples were used in the uniaxial compression tests. The particle size distribution, organic matter content, particle density and soil density were also determined. The load support capacity model for the Oxisol is expressed by the equation $\sigma_{p}=10^{(2,71-1,36 \mathrm{U})}$. All the traffic intensities caused soil compaction in the two depths, where four passes caused highest soil compaction, and as the Forwarder load increased, the soil compaction also increased in the two depths.
\end{abstract}

Keywords:Soil compaction, Preconsolidation pressure and Forest mechanization.

\footnotetext{
${ }^{1}$ Recebido em 13.08.2008 e aceito para publicação em 18.04.2011.

${ }^{2}$ Pesquisador Embrapa Amazônia Oriental - CPATU - Belém/PA, Brasil. E-mail: <arystides@cpatu.embrapa.br>.

${ }^{3}$ Universidade Federal de Lavras, Departamento de Ciência do Solo, Lavras, MG, Brasil. E-mail: <msouzadj@ufla.br>.

${ }^{4}$ Celulose Nipo Brasileira S/A, Cenibra Brasil. E-mail: <fernando.leite@cenibra.com.br>.
} 


\section{INTRODUÇÃO}

As operações mecanizadas de colheita florestal se intensificaram no início da década de 90 do século $\mathrm{XX}$, devido à pressão exercida pelas fábricas de celulose para alcançar maior produtividade impulsionada pelos preços da celulose no mercado internacional. Desde então, a exploração florestal tem causado processo acelerado de degradação da estrutura do solo, devido ao aumento da intensidade do tráfego de máquinas durante as operações de colheita e baldeio da madeira, principalmente quando realizadas em condições inadequadas de umidade (DIAS JUNIOR; PIERCE, 1996; DIAS JUNIOR et al., 1999, 2005; SILVA et al., 2007; , 2009).

O tráfego intensivo de máquinas tem sido o principal responsável pelo aumento da densidade e resistência mecânica do solo, diminuição da porosidade e condutividade hidráulica (HANZA; ANDERSON, 2005; SUZUKI et al., 2007), resultando na compactação do solo e alterando o meio onde o sistema radicular se desenvolve (MARCHÃO et al., 2007). Isso dificulta a penetração das raízes, a extração de água e o crescimento das plantas, afetando seu potencial produtivo (DEDECEK; GAVA, 2005), como a qualidade ambiental em razão do aumento do processo erosivo (KRUMMELBEIN et al., 2008), culminando com a redução da produtividade (REICHERT et al., 2009).

A compactação em solos florestais está relacionada ao uso de máquinas nas operações de colheita e baldeio da madeira (DIAS JUNIOR; PIERCE, 1996; DIAS JUNIOR et al., 1999, 2003; DEDECEK; GAVA, 2005; SILVA et al., 2007), que trafegam várias vezes em uma mesma linha de tráfego ou aleatoriamente na área (RAPER, 2005; HANZA; ANDERSON, 2005; SILVA et al., 2007; SCHAFFER et al., 2008). A carga que essas máquinas transportam pode aplicar pressões aos solos maiores do que a sua capacidade de suporte de carga (DIAS JUNIOR et al., 2005), resultando na compactação.

O tráfego em áreas cultivadas com eucalipto torna-se, portanto, preocupante devido à possibilidade de disseminação da compactação (DIAS JUNIOR et al., 2005, 2007; SILVA et al., 2007), principalmente quando esse tráfego é realizado em condições inadequadas de umidade e por vários ciclos da exploração florestal (RAPER, 2005; HANZA; ANDERSON, 2005; SILVA et al., 2007, 2009).
Em estudos recentes, modelos têm sido utilizados para estimar a capacidade de suporte de carga dos solos, quantificando os níveis de pressões que podem ser aplicados para evitar que a compactação ocorra (DIAS JUNIOR et al., 1999, 2003, 2005, 2007; SILVA et al., 2007) e para monitorar o impacto de cada operação de colheita florestal sobre a estrutura dos solos. Assim, estudos que contemplem o desenvolvimento de modelos de capacidade de suporte de carga que visem à identificação, quantificação e minimização dos efeitos causados pelo baldeio da madeira sobre o solo são importantes para adaptar o manejo do solo e das operações mecânicas, visando à exploração florestal sustentável.

Os objetivos deste estudo foram: a) propor modelo de capacidade de suporte de carga para Latossolo Vermelho-Amarelo no Município de Santa Maria de Itabira, MG, em função da pressão de pré-consolidação e da umidade; e b) determinar, com o uso desses modelos, o efeito da intensidade de tráfego e carga de um Forwarder sobre a compactação do solo durante o transporte da madeira de eucalipto dentro do talhão.

\section{MATERIAL E MÉTODOS}

Este estudo foi conduzido em áreas experimentais da Celulose Nipo-Brasileira S.A. (CENIBRA), no projeto Cuité, localizada no Município de Santa Maria de Itabira, MG, na região de Cocais, apresentando as coordenadas $19^{\circ} 23^{\prime} 11,63^{\prime \prime}$ S e 42 ${ }^{\circ} 54^{\prime} 16,11^{\prime \prime}$ W de Greenwich e altitude de $850 \mathrm{~m}$. O solo na área é um Latossolo VermelhoAmarelo de textura argilosa, relevo declivoso.

As operações de colheita florestal foram realizadas com motosserra e a retirada da madeira, manualmente. Foram demarcadas para cada experimento parcelas de quatro linhas de árvores com 26 árvores na linha com espaçamento de 3 x 2 m, apresentando uma área total de $624 \mathrm{~m}^{2}$, onde dois experimentos foram realizados. No primeiro, avaliou-se o efeito da intensidade de tráfego utilizando o Forwarder autocarregável (marca Valmet, modelo $636 \mathrm{~S}$ ), com tara de 11,9 t, carregado com $9 \mathrm{~m}^{3}$ de madeira (densidade de $480 \mathrm{~kg} \mathrm{~m}^{-3}$ ), trafegando sobre a mesma entrelinha duas, quatro e oito vezes com carga de $3 / 3$ de sua capacidade, o que corresponde a $9 \mathrm{~m}^{3}$ de madeira, e com uma testemunha sem tráfego. No segundo experimento, o Forwarder recebeu cargas correspondentes a 1/3 (3 m $), 2 / 3\left(6 \mathrm{~m}^{3}\right)$ e 3/3 $\left(9 \mathrm{~m}^{3}\right)$ de madeira, trafegando quatro vezes na mesma entrelinha. 
A amostragem foi realizada no mês de novembro de 2004 (21 meses após o tráfego do Forwarder), quando a cultura do Eucalipto urograndis estava sendo conduzida à brotação, e consistiu em coletas de amostras: a) amostras indeformadas, na parcela onde não houve tráfego do Forwarder (testemunha), as quais foram utilizadas na obtenção dos modelos de capacidade de suporte de carga no Latossolo Vermelho-Amarelo; e b) amostras indeformadas coletadas na linha de tráfego, para verificar os efeitos da intensidade de tráfego e carga do Forwarder sobre a estrutura do solo.

Para a obtenção do modelo de capacidade de suporte de carga, foram coletadas 20 amostras indeformadas em cada profundidade (0-3 e 10-13 cm) da áreatestemunha. As amostras foram coletadas usando-se amostrador tipo Uhland com anel volumétrico de 6,40 cm de diâmetro e 2,54 cm de altura, totalizando 40 amostras [20 amostras x 2 profundidades].

Essas amostras foram submetidas ao ensaio de compressão uniaxial de acordo com o método proposto por Bowles (1986), modificado por Dias Junior (1995).

Nos ensaios de compressão uniaxial, as amostras foram previamente saturadas e depois secas ao ar, em laboratório, até atingir as faixas de umidade de 0,05 a $0,47 \mathrm{~kg} \mathrm{~kg}^{-1}$. Após a obtenção dessas umidades, as amostras foram submetidas ao ensaio de compressão uniaxial usando um consolidômetro da marca Boart Longyear, por meio do qual as pressões foram aplicadas com o uso de ar comprimido. As pressões aplicadas a cada amostra obedeceram à seguinte ordem: 25,50 , 100, 200, 400, 800 e 1.600 kPa. Cada pressão foi aplicada até que $90 \%$ da deformação máxima fosse atingida (TAYLOR, 1948) e, depois de essa condição ser atingida, aplicou-se nova pressão.

Após a realização do ensaio de compressão uniaxial, as pressões de pré-consolidação $\left(\sigma_{p}\right)$ foram obtidas na curva de compressão do solo de acordo com Dias Junior e Pierce (1995). As pressões de pré-consolidação foram plotadas em função da umidade, e utilizando-se o software Sigma Plot 8.0 (Jandel Scientific, P.O. Box 7005, San Rafael, CA, USA) foram ajustadas equações matemáticas a esses pontos, que corresponderam aos modelos de capacidade de suporte de carga com os respectivos intervalos de confiança da população a 95\%.

Para avaliar a intensidade de tráfego e carga do Forwarder sobre a estrutura do solo, 10 amostras indeformadas foram coletadas na linha de tráfego em cada profundidade, nas diferentes condições estudadas, totalizando 120 amostras [ 10 amostras x 2 profundidades x 6 condições (2, 4 e 8 passadas e 1/3, 2/3 e 3/3 da carga)]. As amostras indeformadas para a obtenção dos modelos e para a avaliação da intensidade de tráfego foram parafinadas no campo, para evitar alterações da umidade a partir do momento da coleta até o da realização do ensaio de compressão uniaxial em laboratório.

Nessas amostras, os ensaios de compressão uniaxial foram realizados com a umidade na qual as operações com o Forwarder foram realizadas. Obtiveram-se, então, os valores das pressões de pré-consolidação e das umidades que foram representadas em gráfico dos modelos de capacidade de suporte de carga, determinando, assim, os efeitos da intensidade de tráfego e carga do Forwarder sobre a estrutura do solo, de acordo com Dias Junior et al. (2005).

As amostras deformadas foram obtidas pela retirada de material de solo das porções superiores e inferiores dos anéis de amostragem de cada tratamento, obtendose uma amostra composta. Nessas amostras, foram determinados os teores de matéria orgânica (RAIJ; QUAGGIO, 1983), a textura (DAY, 1986), a densidade de partículas e a densidade do solo (BLAKE; HARTGE, 1986).

As análises de regressões foram realizadas com o uso do programa Sigma Plot 8.0 (Jandel Scientific) e as comparações das regressões, feitas segundo o procedimento descrito por Snedecor e Cochran (1989). Os resultados das análises das características físicas do solo (Tabela 1) foram submetidos à análise de variância, sendo a comparação das médias das profundidades realizada pelo teste de Scott-Knott $(\mathrm{p}<0,05)$.

\section{RESULTADOS}

Os dados de textura, matéria orgânica, densidade de partículas e densidade do solo são apresentados na Tabela 1.

Os modelos de capacidade de suporte de carga do Latossolo Vermelho-Amarelo nas profundidades de 0 -3 e 10-13 cm (Figura 1) foram $\sigma_{\mathrm{p}}=10^{(2,70-1,33 \mathrm{U})}$ e $\sigma_{\mathrm{p}}=10^{(2,72-1,40 \mathrm{U})}$, respectivamente, seguindo-se o modelo proposto por Dias Junior (1995) e Dias Junior e Pierce (1996). Os coeficientes de determinação $\left(\mathrm{R}^{2}\right)$ variaram de 0,91 a 0,92 , sendo todos significativos no nível de 1\% (Figura 1).

Revista Árvore, Viçosa-MG, v.35, n.3, p.547-554, 2011 
Tabela 1 - Caracterização física e teor de matéria orgânica de material de Latossolo Vermelho-Amarelo localizado na região de Cocais, coletado nas profundidades de $0-3$ e $10-13 \mathrm{~cm}$.

Table 1 - Physical characterization end content of organic matterfrom an Oxisol located in the Cocais Region sampled at $0-3$ and $10-13 \mathrm{~cm}$ depths.

\begin{tabular}{lcccccc}
\hline Camadas & Argile & Areia & Silte & $\mathrm{MO}^{1}$ & $\mathrm{DP}^{1}$ & $\mathrm{Ds}^{1}$ \\
\hline \multicolumn{5}{c}{} & $\mathrm{cm}$ & \multicolumn{4}{c}{$\mathrm{g} \mathrm{kg}^{-1}$} & \multicolumn{2}{c}{$\mathrm{g} \mathrm{cm}^{-3}$} \\
$0-3$ & $537 \mathrm{~b}$ & $407 \mathrm{a}$ & $56 \mathrm{a}$ & $40 \mathrm{a}$ & $2,50 \mathrm{~b}$ & $0,98 \mathrm{a}$ \\
$10-13$ & $570 \mathrm{a}$ & $367 \mathrm{~b}$ & $63 \mathrm{a}$ & $25 \mathrm{~b}$ & $2,56 \mathrm{a}$ & $0,94 \mathrm{a}$ \\
\hline
\end{tabular}

${ }^{1}: \mathrm{MO}=$ matéria orgânica; $\mathrm{Dp}$ = densidade de partículas; e Ds densidade do solo. Médias nas colunas com a mesma letra não diferem entre si, pelo teste de Scott-Knott a 5\% de probabilidade.

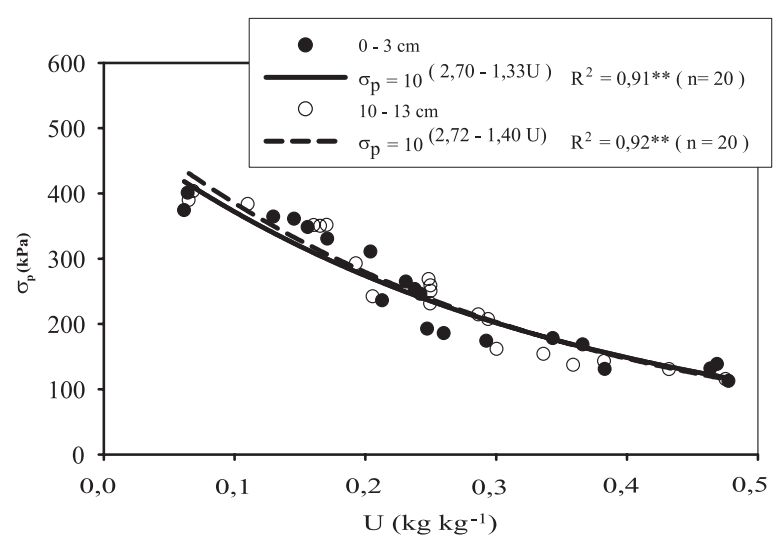

Figura 1 - Modelos da capacidade de suporte de carga do Latossolo Vermelho-Amarelo nas profundidades de $0-3$ e $10-13 \mathrm{~cm}$.

Figure 1 - Models of load support capacity for the Oxisol at $0-3$ and $10-13 \mathrm{~cm}$ depths.

Os modelos de capacidade de suporte de carga foram homogêneos, de acordo com o procedimento proposto por Snedecor e Cochran (1989), indicando mesma capacidade de suporte de carga nas profundidades de 0-3 e 10-13 cm. Em razão disso, nova equação de regressão foi ajustada, considerando-se todos os valores de pressão de pré-consolidação e de umidade, e assim foi obtido um único modelo de capacidade de suporte de carga, $\mathrm{s}_{\mathrm{p}}=10^{(2,71-1,36 \mathrm{U})}$, com $\mathrm{R}^{2}$ significativo a $1 \%$ e igual a 0,92 , nas duas profundidades do Latossolo Vermelho-Amarelo (Figura 2).

Para avaliar a intensidade de tráfego e carga do Forwarder sobre a estrutura do LVA, foram inseridos no modelo de capacidade de suporte de

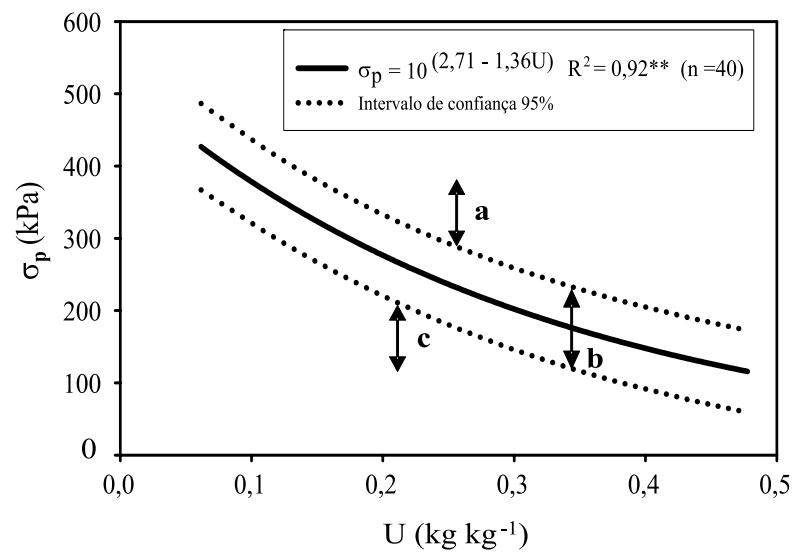

Figura 2-Modelo da capacidade de suporte de carga do Latossolo Vermelho-Amarelo nas profundidades de 0-3 e 10$13 \mathrm{~cm}$. As regiões indicadas pelas letras “a”, “b” e “c” foram estipuladas de acordo com Dias Junior et al. (2005).

Figure 2 - Models of load support capacity for the Oxisol at 0 -3 and 10-13 cm depths. The regions indicated by the letters " $a$ ", " $b$ " and " $c$ " were set according to Dias Junior et al. (2005).

carga (Figura 2) os valores das pressões de préconsolidação com as respectivas umidades, obtendo-se as Tabelas 2 e 3, respectivamente, de acordo com os critérios usados por Dias Junior et al. (2005). Assim, dividiu-se a Figura 2 em três regiões.

As regiões consideradas são: a) aquela onde os valores da pressão de pré-consolidação determinados após o tráfego são maiores que o limite superior do intervalo de confiança, sendo considerada a região com compactação do solo adicional; b) região onde os valores da pressão de pré-consolidação determinados após o tráfego estão entre os limites de confianças inferior e superior, considerada como região sem compactação, mas com tendência a isso. Embora as amostras de solo situadas aí não tenham sido compactadas, essa região indica que as amostras podem vir a ser compactadas nas próximas operações de colheita, se as pressões aplicadas forem maiores que o limite superior do intervalo de confiança; e c) região onde os valores da pressão de pré-consolidação determinada após o tráfego são menores que o limite inferior do intervalo de confiança, considerada a região sem compactação. 
Tabela 2 - Valores médios de umidade e pressão de pré-consolidação e classificação das amostras de solo em porcentagem, de acordo com cada região “a”, "b” e "c" da Figura 2, usando os valores de pressão de pré-consolidação determinados após as operações do Forwarder em material de solo de Latossolo Vermelho-Amarelo, nas profundidades de 0-3 e 10-13 cm, localizado na região de Cocais, utilizando os critérios de Dias Junior et al. (2005).

Table 2 - Average moisture and preconsolidation pressure and classification of soil samples in percentage, according to the zone " $a$ ", " $b$ " and " $c$ " in Figure 2, using the values of preconsolidation pressure determined after the Forwarder operations for a Oxisol for 0-3 and 10-13 cm depths located in the Cocais Region using the criteria for Dias Junior et al. (2005).

\begin{tabular}{|c|c|c|c|c|c|c|}
\hline \multicolumn{7}{|c|}{ Efeito da Intensidade de Tráfego Forwarder } \\
\hline \multirow{4}{*}{ Região } & \multicolumn{6}{|c|}{ Profundidade de $0-3 \mathrm{~cm}$} \\
\hline & \multicolumn{2}{|c|}{2 Passadas $\left(9 \mathrm{~m}^{3}\right)$} & \multicolumn{2}{|c|}{4 Passadas $\left(9 \mathrm{~m}^{3}\right)$} & \multicolumn{2}{|c|}{8 Passadas $\left(9 \mathrm{~m}^{3}\right)$} \\
\hline & \multirow{2}{*}{$\begin{array}{c}\mathrm{U} \\
\mathrm{kg} \mathrm{kg}^{-1} \\
0,22^{(1)}\end{array}$} & \multirow{2}{*}{$\begin{array}{c}\sigma_{\mathrm{p}} \\
\mathrm{kPa} \\
295^{(1)}\end{array}$} & \multirow{2}{*}{$\begin{array}{c}\mathrm{U} \\
\mathrm{kg} \mathrm{kg}^{-1} \\
0,23^{(1)}\end{array}$} & \multirow{2}{*}{$\begin{array}{c}\sigma_{\mathrm{p}} \\
\mathrm{kPa} \\
340^{(1)}\end{array}$} & \multirow{2}{*}{$\begin{array}{c}\mathrm{U} \\
\mathrm{kg} \mathrm{kg}^{-1} \\
0,23^{(1)}\end{array}$} & $\begin{array}{c}\sigma_{\mathrm{p}} \\
\mathrm{kPa}\end{array}$ \\
\hline & & & & & & $303^{(1)}$ \\
\hline & & & \multicolumn{2}{|c|}{$\%$} & \\
\hline a - Compactação & \multicolumn{2}{|c|}{30} & \multicolumn{2}{|c|}{100} & \multicolumn{2}{|c|}{60} \\
\hline b - Sem compactação, mas com risco de compactar & \multicolumn{2}{|c|}{70} & \multirow{2}{*}{\multicolumn{2}{|c|}{$\begin{array}{l}0 \\
0\end{array}$}} & \multicolumn{2}{|c|}{40} \\
\hline \multirow[t]{2}{*}{ c - Sem compactação } & \multicolumn{2}{|c|}{0} & & & & \\
\hline & \multicolumn{6}{|c|}{ Profundidade de $10-13 \mathrm{~cm}$} \\
\hline \multirow[t]{3}{*}{ Região } & \multicolumn{2}{|c|}{2 Passadas $\left(9 \mathrm{~m}^{3}\right)$} & \multicolumn{2}{|c|}{4 Passadas $\left(9 \mathrm{~m}^{3}\right)$} & \multicolumn{2}{|c|}{8 Passadas $\left(9 \mathrm{~m}^{3}\right)$} \\
\hline & $\begin{array}{c}\mathrm{U} \\
\mathrm{kg} \mathrm{kg}^{-1} \\
023^{(1)}\end{array}$ & $\begin{array}{c}\sigma_{\mathrm{p}} \\
\mathrm{kPa} \\
360^{(1)} \\
\end{array}$ & $\begin{array}{c}\mathrm{U} \\
\mathrm{kg} \mathrm{kg}^{-1} \\
0,22^{(1)}\end{array}$ & $\begin{array}{c}\sigma_{\mathrm{p}} \\
\mathrm{kPa} \\
346^{(1)} \\
\end{array}$ & $\begin{array}{c}\mathrm{U} \\
\mathrm{kg} \mathrm{kg}^{-1} \\
0,22^{(1)}\end{array}$ & $\begin{array}{c}\sigma_{\mathrm{p}} \\
\mathrm{kPa} \\
334^{(1)} \\
\end{array}$ \\
\hline & \multicolumn{6}{|c|}{$\%$} \\
\hline a - Compactação & \multicolumn{2}{|c|}{100} & \multicolumn{2}{|c|}{80} & \multicolumn{2}{|c|}{80} \\
\hline b - Sem compactação, mas com risco de compactar & \multicolumn{2}{|c|}{0} & \multicolumn{2}{|c|}{20} & \multicolumn{2}{|c|}{20} \\
\hline c - Sem compactação & \multicolumn{2}{|c|}{0} & \multicolumn{2}{|c|}{0} & \multicolumn{2}{|c|}{0} \\
\hline
\end{tabular}

${ }^{(1)}$ Médias de 10 repetições.

\section{DISCUSSÃO}

Os resultados apresentados na Tabela 2 indicam que todas as intensidades de tráfego na área do estudo causaram compactação. O número de duas passadas na mesma entrelinha pelo Forwarder promoveu maior compactação na profundidade de $10-13 \mathrm{~cm}$ que quatro e oito na mesma entrelinha em razão, provavelmente, do maior teor de argila e menor teor de matéria orgânica nessa profundidade. Esses resultados corroboram os obtidos por Hanza e Anderson (2005), que em revisão de literatura de trabalhos relacionados com compactação do solo publicados nos últimos 15 anos e, Seixas e Souza (2007), em um estudo na Estação experimental de Itatinga, SP, pertencente à Escola Superior de Agricultura “Luiz de Queiroz” em um solo arenoso cultivado com eucalipto, observaram que a maior parte da compactação do solo ocorre na primeira passada de uma roda e que esta pode aumentar com o número de passadas. O número de passadas igual a 4 foi o que causou maior compactação na profundidade de $0-3 \mathrm{~cm}$, identificando que, com o aumento do número de passadas, os teores de argila e matéria orgânica do solo em estudo não influenciaram a compactação do solo em profundidade. Já o número de passadas igual a 8 causou menor compactação do que o número de passadas igual a 4 na profundidade de $0-3 \mathrm{~cm}$ e do que o número de passadas igual a 2 na profundidade de $10-13 \mathrm{~cm}$. Esses resultados são justificados pelo fato de que, nessas situações, os níveis de pressões aplicadas pelo Forwarder foram maiores do que a capacidade de suporte de carga do solo, o que promoveu destruição de sua estrutura, com consequente redução nas pressões de pré-consolidação. Esses resultados corroboram os encontrados por Silva et al. (2006), Lopes et al. (2006) e Dedecek e Gava (2005), que observaram camadas compactadas em profundidade e, ainda, que, à medida que a intensidade de tráfego aumentava, a compactação do solo era mais evidenciada na profundidade de $0-3 \mathrm{~cm}$, desde que essa intensidade não ultrapassasse a capacidade-suporte de carga do solo.

Quando a carga do Forwarder foi aumentada de $3 \mathrm{~m}^{3}$ para $6 \mathrm{~m}^{3}$ e para $9 \mathrm{~m}^{3}$ (Tabela 3), as porcentagens de amostras compactadas foram de 60, 80 e 90\%, 
Tabela 3 - Valores médios de umidade e pressão de pré-consolidação e classificação das amostras de solo em porcentagem, de acordo com cada região “a”, "b” e “c” da Figura 2, usando os valores de pressão de pré-consolidação determinados após quatro passadas do Forwarder com diferentes cargas, em um Latossolo Vermelho-Amarelo, nas profundidades de 0-3 e 10-13 cm, localizado na região de Cocais, utilizando os critérios de Dias Junior et al. (2005).

Table 3 - Average moisture and preconsolidation pressure and classification of soil samples in percentage, according to the zone " $a$ ", " $b$ " and " $c$ " in Figure 2, using the values of preconsolidation pressure determined after four Forwarder of the past with different charges for an Oxisolfor 0-3 and 10-13 cm depths located in the Cocais Region using the criteria for Dias Junior et al. (2005).

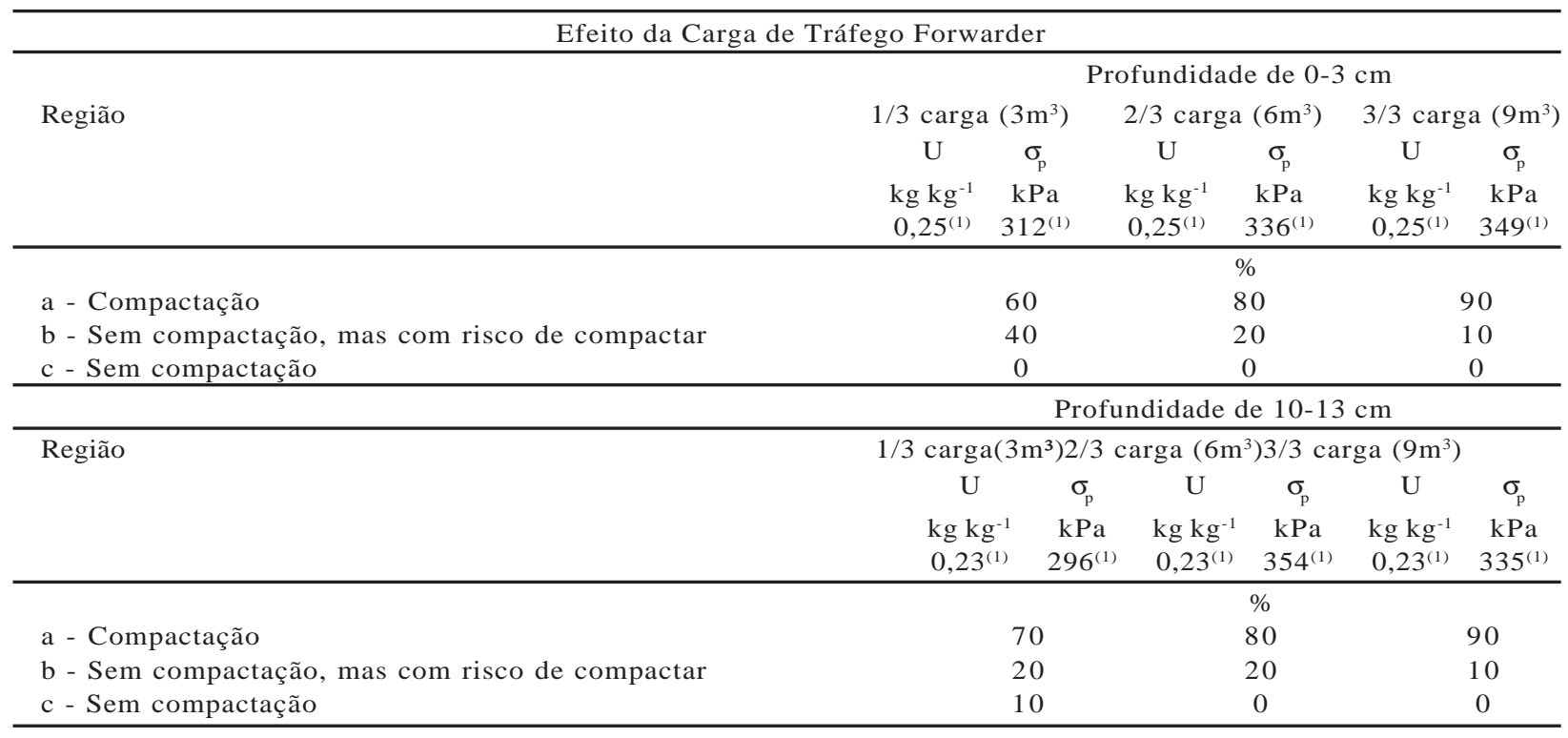

${ }^{(1)}$ Médias de 10 repetições.

respectivamente, na profundidade de $0-3 \mathrm{~cm}$ e de 70,80 e $90 \%$ na profundidade de $10-13 \mathrm{~cm}$, respectivamente. Esses resultados indicam que, com o aumento da carga, ocorreu incremento da compactação nas duas profundidades. Esses resultados discordam dos obtidos por Çarman (2002), em um estudo realizado em um latossolo na Universidade de Selçuk, na Turquia, segundo o qual o aumento da carga não eleva, necessariamente, a compactação do solo, devido ao aumento da superfície de contato pneu/solo, resultando na redistribuição da carga no solo. No entanto, os dados corroboram os resultados obtidos por Silva et al. (2007), em um Latossolo Vermelho-Amarelo localizado no Município de Santa Maria de Itabira, MG, onde observaram que, após 441 dias sem nenhum tráfego na área, houve decréscimo significativo na densidade do solo onde o Forwarder trafegou carregado com $4 \mathrm{~m}^{3}$ de madeira, indicando que com menores cargas podem ocorrer resiliência em relação à compactação do solo ao longo do tempo.

Revista Árvore, Viçosa-MG, v.35, n.3, p.547-554, 2011

\section{CONCLUSÕES}

O modelo de capacidade-suporte de carga do LVA é expresso pela equação $\sigma_{\mathrm{p}}=10^{(2,71-1,37 \mathrm{U})}$.

A intensidade de tráfego de quatro passadas na mesma entrelinha foi a que causou maior compactação do solo.

À medida que elevou a carga do Forwarder, a compactação do solo aumentou nas duas profundidades.

\section{REFERÊNCIAS}

BLAKE, G. R.; HARTGE, K. H. Particle density. In: KLUTE, C. (Ed.). Methods of soil analysis. Physical and mineralogical methods. 2.ed. Madison: American Society Agronomy, 1986. Part 1. p.377-382. (Agronomy Monograph, 9)

BOWLES, J. E. Engieneering properties of soils and their measurements. 3.ed. Auckland: McGraw-Hill, 1986. 218p. 
ÇARMAN, K. Compaction characteristics of towed wheels on clay loam in a soil bin. Soil \& Tillage Research, v.65, n.1, p.37-43, 2002.

DAY, P. R. Particle fractionation and particle size analysis. In: KLUTE, C. A. (Ed.). Methods of soil analysis. Physical and mineralogical methods. 2.ed. Madison: American Society Agronomy, 1986. Part 1. p.545-567. (Agronomy Monograph, 9)

DIAS JUNIOR, M. S.; PIERCE, F. J. A simple procedure for estimating preconsolidation pressure from soil compression curves. Soil Technology, v.8, n.2, p.139-151, 1995.

DIAS JUNIOR, M. S.; PIERCE, F. J. Revisão de Literatura. O processo de compactação do solo e sua modelagem. Revista Brasileira de Ciência do Solo, v.20, n.2, p.175-182, 1996.

DIAS JUNIOR, M. S. Compactação do solo. In: NOVAIS, R. F.; ALVAREZ V., H. V. \& CHAEFER, C. E. G. R., (Ed.) Tópicos em ciência do solo. Viçosa, MG: Sociedade Brasileira de Ciência do Solo, 2000. v.1. p.55-94.

DIAS JUNIOR, M. S. et al. Avaliação quantitativa da sustentabilidade estrutural dos solos em sistemas florestais na região de Aracruz-ES. Revista Árvore, v.23, n.3, p.371-380, 1999.

DIAS JUNIOR, M. S. et al. Avaliação quantitativa da sustentabilidade estrutural de um Latossolo Amarelo cultivado com eucalipto na Região de Peçanha - MG. Revista Árvore, v.27, n.3, p.343-349, 2003.

DIAS JUNIOR, M. S. et al. Traffic effects on the soil preconsolidation pressure due to eucalyptus harvest operations. Scientia Agricola, v.62, n.3, p.248-255, 2005.

DEDECEK, R. A.; GAVA, J. L. Influência da compactação do solo na profundidade da rebrota de eucalipto. Revista Árvore, v.29, n.3, p.383-390, 2005

HAMZA, M. A.; ANDERSON, W. K. Soil compaction in cropping systems a review of the nature, causes and possible solutions. Soil \& Tillage Research, v.82, n.2, p.121-145, 2005.
KUMMELBEIN, J.; PETH, S.; HORN, R.

Determination of pre-compression stress of a variously grazed steppe soil under static and cyclic loading. Soil and Tillage Research, v.99, n.2, p.139-148, 2008.

LOPES, S. E. et al. Compactação de um solo de uso florestal submetido ao tráfego de arraste de madeira. Revista Árvore, v.30, n.3, p.369-376, 2006.

MARCHÃO, R. L. et al. Qualidade física de um Latossolo Vermelho sob sistemas de integração lavoura-pecuária no Cerrado. Pesquisa Agropecuária Brasileira, v.4, n.6, p.873882, 2007.

RAIJ, B. van; GUAGGiO, J. A. Métodos de análise de solo para fins de fertilidade. Campinas: Instituto Agronômico de Campinas, 1983. 16p. (Circular, 63)

REICHERT, J. M. et al. Reference bulk density and critical degree-of-compactness for no-till crop production in subtropical highly weathered soils. Soil and Tillage Research, v.102, n.2, p.242-254, 2009

RAPER, R. L. Agricultural traffic impacts on soil. Journal of Terramechanics, v.42, n.3/4, p.259-280, 2005.

SEIXAS, F.; SOUZA, C. R. Avaliação e efeito da compactação do solo, devido à freqüência de tráfego, na produção de madeira de eucalipto. Revista Árvore, v.31, n.6, p.1047-1052, 2007.

SCHAFFER, B. et al. Soil and macro-pores under uniaxial compression: I mechanical stability of repacked soil and deformation of different types of macro-pores. Geoderma, n.1/2, p.183-191, 2008.

SILVA, A. R. et al. Modelagem da Capacidade suporte de carga e quantificação dos efeitos das operações mecanizadas em um Latossolo amarelo cultivado com cafeeiros. Revista Brasileira Ciência do Solo, v.31, n.2, p.207-216, 2006.

SILVA, S. R. et al. Alterações do solo influenciadas pelo trafego e carga de um forwarder nas entrelinhas de uma floresta de eucalipto. Revista Brasileira de Ciência do Solo, v.31, n.2, p.371-377, 2007.

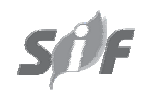

Revista Árvore, Viçosa-MG, v.35, n.3, p.547-554, 2011 
SILVA, A. R.; DIAS JUNIOR, M. S.; LEITE, F.P. Camadas de resíduos florestais e pressão de preconsolidação de dois latossolos. Pesquisa Agropecuária Brasileira, V. 42, n.1, p.89-93, 2007.

SILVA, R. B. et al. Estimation and evaluation of dynamic properties as indicators of changes on soil structure in surgarcane fields of São Paulo state - Brazil. Soil and

Tillage Research, Amsterdam, v.103, n.2, p.265-270, 2009.
SNEDECOR, G. W. \& COCHRAN, W. G.

Statistical methods. 8.ed. Ames: Iowa State University Press, 1989. 503p.

SUZUKI, L.E. A. S. et al. Grau de compactação, propriedades físicas e rendimento de culturas em Latossolo e Argissolo. Pesquisa Agropecuária Brasileira, v.42, n.8, p.1159-1167, 2007.

TAYLOR, D. W. Fundamentals of soil mechanics. New York: John Wiley \& Sons, 1948. $700 \mathrm{p}$. 